\title{
Chemokine-Like Factor 1 (CLFK1) Is Over-Expressed in Patients with Atopic Dermatitis
}

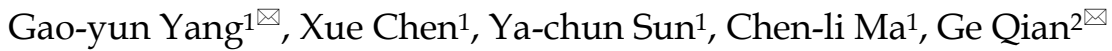 \\ 1. Department of Dermatology, Beijing Friendship Hospital, Capital Medical University, Beijing 100050, China; \\ 2. Department of Dermatology and Rheumatology, the 2nd Affiliated Hospital of Kunming Medical University, Kunming 650101, China.
}

$\triangle$ Corresponding author: Gao-yun Yang, M.D., Ph.D., Department of Dermatology, Beijing Friendship Hospital, Capital Medical University, No. 95 Yong An Road, Xi Cheng District, Beijing 100050, China. Tel: +86 13520713158; Fax: +86 10 63139209; Email: gaoyun@ccmu.edu.cn Or Ge Qian, M.D., Ph.D., Department of Dermatology and Rheumatology, the 2nd Affiliated Hospital of Kunming Medical University, No. 374 Dianmian Road, Kunming Yunnan, 650101. Tel: +86 871 65357115; Fax: +86 871 65352087; Email: drqiange@sina.com.

() Ivyspring International Publisher. This is an open-access article distributed under the terms of the Creative Commons License (http://creativecommons.org/ licenses/by-nc-nd/3.0/). Reproduction is permitted for personal, noncommercial use, provided that the article is in whole, unmodified, and properly cited.

Received: 2013.03.20; Accepted: 2013.07.24; Published: 2013.08.13

\begin{abstract}
Background: Human chemokine-like factor 1 (CKLF1), a recently discovered chemokine, has a broad spectrum of biological functions in immune-mediated diseases. It is highly expressed on Th2 lymphocytes and is a functional ligand for human CCR4. CKLF1 has a major role in the recruitment and activation of leucocytes, which plays an important role in the pathogenesis of allergic diseases. The present study was designed to determine the expression of CKLF1 in skin and serum in patients with atopic dermatitis (AD).

Methods: The CKLF1 protein expression in skin lesion was analyzed by immunohistochemistry and ELISA. The mRNA expression of CKLF1 in skin lesion was detected by Real-time PCR. The serum levels of CKLF1, IgE, eotaxin, IL-4, IL-5, and IL-13 were measured by ELISA.

Results: Histopathological changes in the skin of $A D$ patients showed local inflammation with epidermal thickening and significant inflammatory cellular infiltration. Immunohistochemistry results demonstrated that CKLF1-staining positive cells were located in the epidermal and dermis, and that the CKLF1 expression in AD patients was significantly higher than that in normal control. The CKLF1 mRNA expression in AD patients was significantly higher than that in healthy controls. Serum CKLF1 and IgE levels were significantly increased in AD patients, as were the serum levels of IL-4, IL-5, IL-13 and eotaxin.

Conclusions: Both CKLF1 protien and mRNA levels are overexpressed in the skin lesion of AD patients, along with an increase in serum CKLF1 level, indicating that CKLF1 may play an important role in the development of atopic dermatitis.
\end{abstract}

Key words: atopic dermatitis (AD), chemokine-like factor 1 (CKLF1).

\section{Introduction}

Atopic dermatitis (AD) is a chronically relapsing, inflammatory skin disease characterized by pruritic and eczematous skin lesions and the prevalence of $\mathrm{AD}$ is steadily increasing. Most AD patients have a personal or family history of allergic diseases such as asthma and allergic rhinitis [1]. AD has affected up to
$3 \%$ of adults and $25 \%$ of children [2]. Infants with $\mathrm{AD}$ have an increased tendency to develop asthma and allergic rhinitis later in life [3]. Unfortunately, our understanding of $\mathrm{AD}$ pathogenesis and mediators involved in its onset and progression is rather limited, hampering the discovery and development to effica- 
cious therapeutic agents for this disease. Up to today, the two major drug classes recommended for the treatment of $\mathrm{AD}$ are steroids and antihistamines, which are generally considered as non-specific anti-inflammatory agents.

Chemokines and their receptors play a vital role in the pathogenesis of allergic diseases [4,5]. In the development and progression of $\mathrm{AD}$, the Th2-predominant inflammatory response can lead to IgE production. When IgE-binding mast cells and basophils are exposed to subsequent allergens, the release of chemical mediators often causes allergic reactions [6]. Chemokine-like factor 1 (CKLF1) is a recently discovered cytokine belonging to the chemokine-like factor protein family [7]. In vitro studies demonstrate that CKLF1 has broad chemotactic activities on neutrophils, lymphocytes, monocytes, and nerve cells [7]. There are increasing evidences that demonstrate CKLF1 may play an important role in the pathogenesis of allergic diseases. CKLF1 is up-regulated in lung tissues from asthma patients [8]. In addition, intramuscular injection of CKLF1 plasmid DNA into BALB/c mice causes dramatic pathological changes in the lungs that are similar to those observed in asthma patients [8]. Although CKLF1 has been shown to be a regulator in pathogenesis of asthma and allergic rhinitis [9], its role in AD remains unclear. Therefore, we hypothesized that CKLF1 might play a role in the pathogenesis of AD. To test that, we analyzed the expression of CKLF1 in skin and serum of AD patients in the present study. To investigate its possible roles in AD development and progression, we also detected the expression of some important cytokine in AD patients. To our best knowledge, this is the first study to do so. We believe that targeting CKLF1 may provide a new avenue of therapeutic approach for AD.

\section{Materials and methods}

\section{Patients, specimens and tissue sample treat- ment}

This clinical study protocol was reviewed and approved by the Bioethical Commission of Beijing Friendship Hospital. Informed consent was obtained from all of the patients and controls prior to the study. We enrolled 20 adult patients with AD (11 female and 9 male), age from 18 to 38 (the mean age: 27.6) and 10 volunteers ( 5 female and 5 male) in good health as a control panel. The diagnosis of $\mathrm{AD}$ was made and confirmed by Hanifin and Rajka's criteria [10]. Prior to the study, none of $\mathrm{AD}$ patients or controls received any drugs that might affect the results of the performed assays. Skin specimens were collected by a 4-mm disposable biopsy punch (Miltex Inc., York,
USA) after local anaesthesia. In AD patients, biopsy specimens were collected from skin lesions. Control specimens were collected from health volunteers. Two specimens were collected from each individual: one was put into $1 \mathrm{ml}$ of PBS for generating skin homogenate and subsequent ELISA and the other was cut into two parts, one part being immediately immersed in RNA extraction solution and used for subsequent real-time PCR analysis and the other part being prepared for histopathological analysis. A standard ELISA protocol was used to quantify the total amount of CKLF1 in supernatants of the skin homogenate (R\&D Systems).

\section{Real-time quantitative PCR}

The total RNA was extracted from human skin specimens and was reverse-transcribed by a Reverse Transcription Kit (Invitrogen). The real-time PCR amplification was performed using an Mx3000 Multiplex Quantitative PCR System (Stratagene Corp, La Jolla, CA), the primers for human CKLF1 were as follows: forward, 5'-TCGCTTCGCAGAACCTACTCA-3' , reverse, 5'-TATTTTCGGCTGCACGTTATCC-3'. All amplification reactions were conducted for 45 cycles in duplicate (an initial stage of $2 \mathrm{~min}$ at $95^{\circ} \mathrm{C}$, followed by a three-step cycle of $20 \mathrm{~s}$ at $95^{\circ} \mathrm{C}, 25 \mathrm{~s}$ at $59^{\circ} \mathrm{C}$ and $30 \mathrm{~s}$ at $72{ }^{\circ} \mathrm{C}$ ). The results were expressed as relative quantification units (fold change). Glyceraldehyde-3-phosphate dehydrogenase (GAPDH) was applied to each sample as an internal control in order to normalize CKLF1 expression levels.

\section{Quantitation of CKLF1, IgE, IL-4, IL-5, IL-13 and eotaxin in the serum and/or skin tissue}

The CKLF1 levels in serum and supernatant of the skin homogenates were measured by ELISA following the manufacturer's instruction (R\&D Systems, Minneapolis, USA). The serum levels of IgE, IL-4, IL-5, IL-13, and eotaxin were measured by ELISA in accordance with manufacturer's instruction (R\&D Systems and BioLegend). The ELISA kits had a detection limit of $4 \mathrm{pg} / \mathrm{ml}$. Briefly, $100 \mu \mathrm{l}$ of each diluted sample was applied in triplicate on 96-well plates pre-coated with capture antibody for $2 \mathrm{~h}$, followed by incubation with detection antibody for $1 \mathrm{~h}$ and avidin-HRP for $30 \mathrm{~min}$. The plates were then developed using 3, 3', 5, 5'-tetramethylbenzidine and terminated by $2 \mathrm{~N} \mathrm{H}_{2} \mathrm{SO}_{4}$. The OD value was then recorded at 450 $\mathrm{nm}$.

\section{Histopathological and immunohistochemical examination}

Human skin specimens were fixed in $10 \%$ formalin solution for $48 \mathrm{~h}$. The fixed specimens were then cut transversely into $5-\mu \mathrm{m}$ sections. Each section 
was embedded in paraffin and stained with haematoxylin and eosin. For immunohistochemical staining, an indirect avidin/biotinylated alkaline phosphatase complex method was used, rabbit anti-human CKLF1 antibody was diluted into $5 \mathrm{ng} / \mathrm{ul}$ (kind gift from Prof. Wenling Han, Peking University, Beijing, China) and used as primary antibody for CKLF1 staining.

\section{Statistical Analysis}

All statistical analyses were accomplished by Student's t-test using the software SPSS 19.0. The results were presented as mean \pm standard deviation $(\bar{x}$ $\pm \mathrm{s})$. Values of $\mathrm{P}<0.05$ were considered statistically significant.

\section{Results}

\section{The CKLF1 levels in both serum and skin of the AD patients were significantly elevated}

We first detected the CKLF1 levels in skin homogenates and in sera of the AD patients. As shown

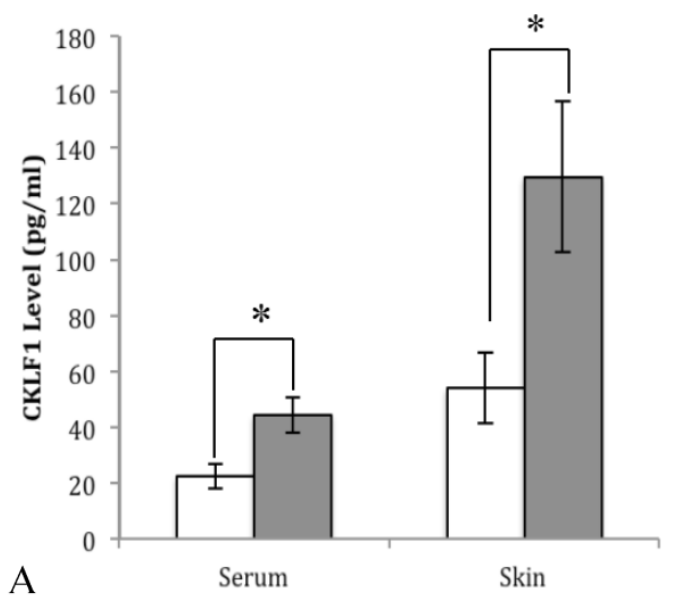

in Figure $1(\mathrm{~A})$, the CKLF1 levels were significantly increased in $\mathrm{AD}$ patients than that in normal controls.

\section{The CKLF1 mRNA was overexpressed in AD patient's skin lesions}

The expression of CKLF1mRNA in skin tissues from $\mathrm{AD}$ patients and normal controls was examined by real time RT-PCR. As shown in Figure 1 (B), the CKLF1 mRNA expression levels were significantly elevated in $\mathrm{AD}$ group, compared with the controls.

The serum levels of eotaxin, IL-4, IL-5, IL-13 and $\mathrm{IgE}$ in $\mathrm{AD}$ patients were significantly elevated

In order to prove the involvement of eotaxin, IL-4, IL-5, IL-13 and IgE in AD, their serum levels were measured in both patients and controls. As shown in Figure 2 (A), the levels of eotaxin, IL-4, IL-5 and IL-13 in AD patients were significantly higher than that in normal controls. As shown in Figure 2 (B), the elevated levels of IgE in $\mathrm{AD}$ patients were observed in comparison with that in normal controls.

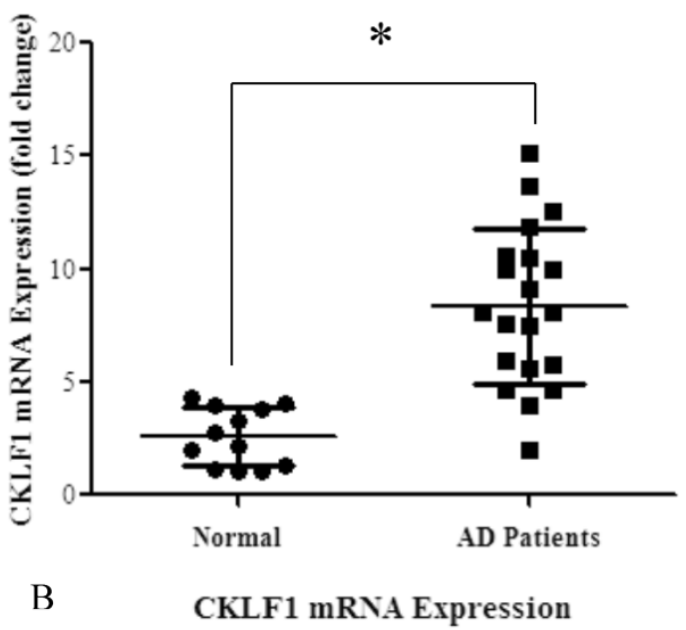

Fig 1. Expression of CKLF1 in AD patients. (A): The CKLF1 protein levels in sera and skin of AD patients were significantly higher than that in the controls. $(* \mathrm{P}<0.05)(B):$ The CKLF1 mRNA levels were significantly higher in $A D$ patients than that in the control group. $(* \mathrm{P}<0.05)$.
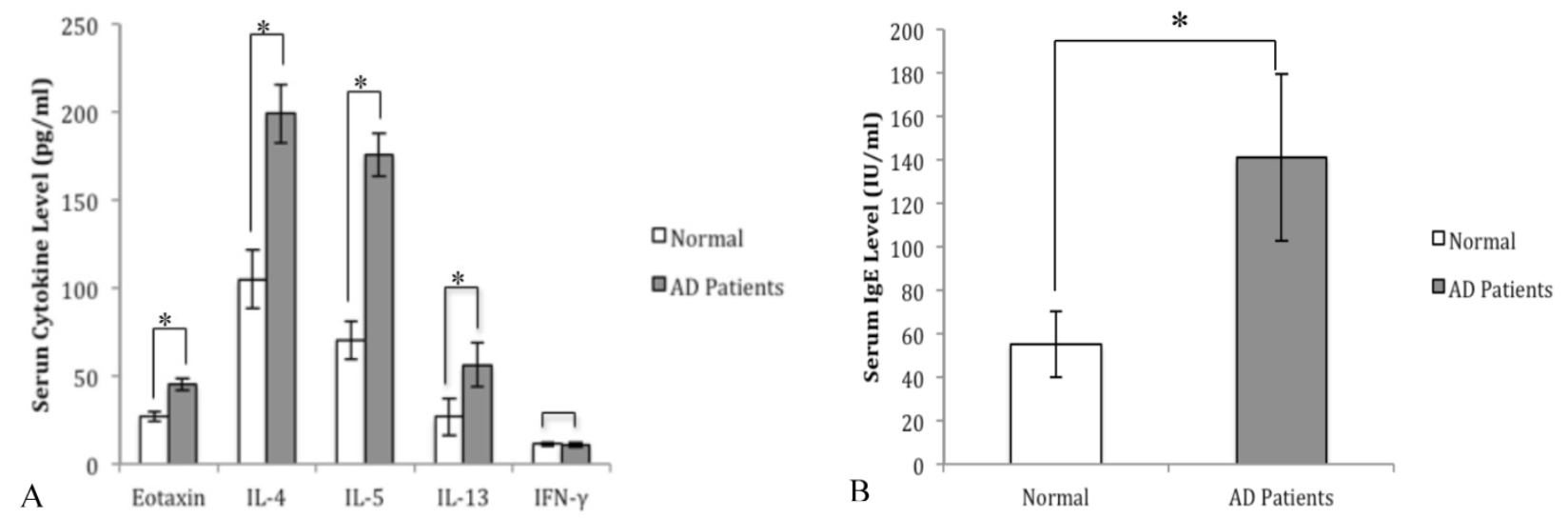

Fig 2. The serum levels of eotaxin-1, IL-4, IL-5, and IL-13 in AD patients were significantly higher than that in the controls $(A)(* P<0.05)$. The serum level of $\lg \mathrm{E}$ in $\mathrm{AD}$ patients was significantly higher than that in the control group $(B)(* \mathrm{P}<0.05)$. 


\section{The CKLF1 overexpression is demonstrated by Histopathological and immunohistochemi- cal analyses}

In skin lesions of $\mathrm{AD}$ patients, the pathologic changes showed slight psoriasiform hyperplasia of the epidermis with slight hyperkeratosis and some parakeratosis. There was more lymphocytic infiltration with admixture of eosinophils and mastocytes in the AD samples than in the control group, which were consistent with histopathological characteristics of $\mathrm{AD}$ [11]. The results of immunohistochemical staining for CKLF1 in the skin indicated that brown granular staining of CKLF1 in cytoplasma and nuclei was distributed on the keratinocytes of granular layer and basal layer in both patients and volunteers (Figure 3). The skin lesion in AD patients showed more staining positive cells and higher intensity in the epidermal and dermis than that in normal skin. The CKLF1 staining positive cells in upper dermis of the AD patients were detected in lymphocytes and other infiltrated cells. There was an obvious increase in CKLF1 staining cells and higher intensity in the dermis of AD patients than normal controls (Figure 3).

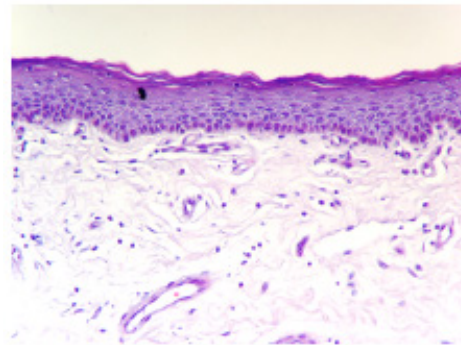

(Normal 1, HE) x 200

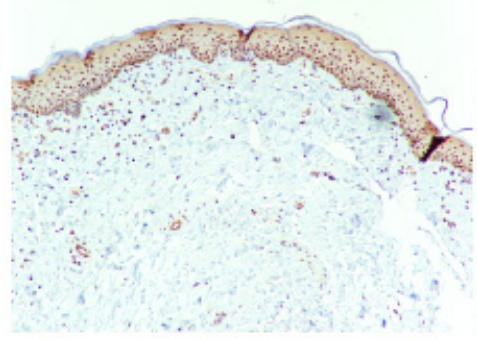

(Normal 2, CKLF1 IHC) x 100

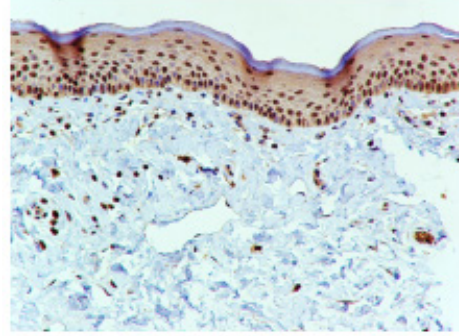

(Normal 3, CKLF1 IHC) x 200

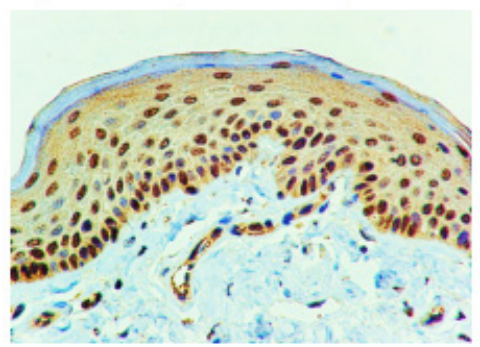

(Normal 4, CKLF1 IHC) x 400

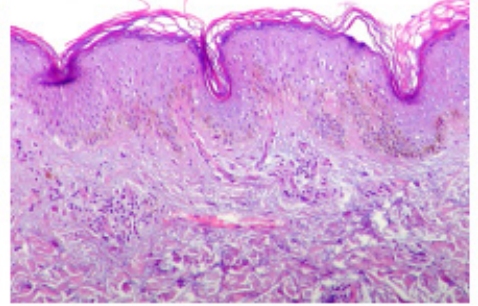

$(\mathrm{AD} 1, \mathrm{HE}) \times 200$

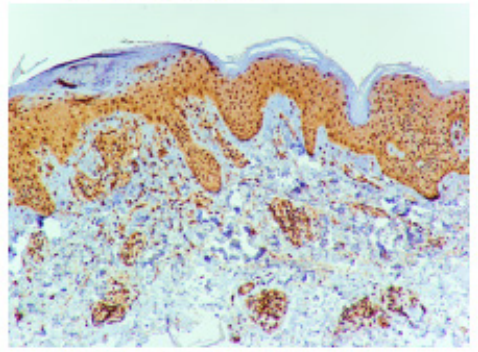

$(\mathrm{AD} 2, \mathrm{CKLF} 1 \mathrm{IHC}) \times 100$

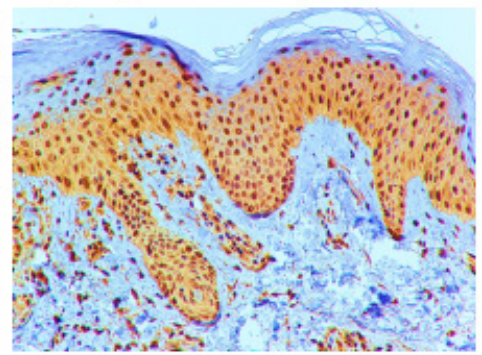

$(\mathrm{AD} 3, \mathrm{CKLF} 1 \mathrm{IHC}) \times 200$

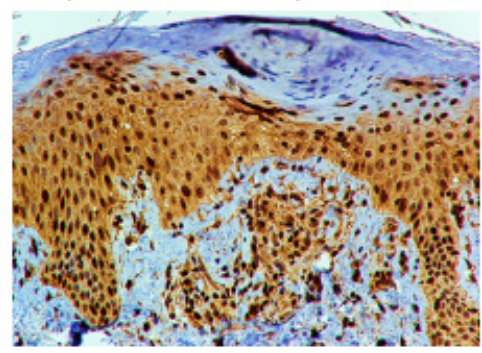

(AD4, CKLF $1 \mathrm{IHC}) \times 400$

Fig 3. Histopathological and immunohistochemical analysis of $A D$ skin lesions and controls. Top panel (Normal $1, A D 1)$ as $H E$ staining and the rest as CKLF1 IHC, the CKLF1 staining positive cells could be found in normal skin (Normal 2, Normal 3, Normal 4) and in skin lesion (AD2, AD3, AD4) of AD patients. However, the skin lesions in AD patients showed much more CKLF1-positive cells with higher intensity in the epidermal and dermis than that in normal skins. 


\section{Discussion}

Atopic dermatitis is a Th2 lymphocyte-dominant inflammatory disease and characterized by chronic and relapsing inflammatory dermatitis. The natural history and sequence of events in $\mathrm{AD}$ pathogenesis have not been well characterized. The trigger point of $\mathrm{AD}$ is believed to be closely related to the imbalance of Th1/Th2-type response [12]. The Th1 and Th2 cytokines are expressed by a variety of cells and their functions are different, suggesting that an imbalance in the expression of Th1 and Th2 cytokines may dictate the different immunopathological responses.

Following antigen stimulation, $\mathrm{T}$ cells can develop into Th2 cells that secrete IL-4, IL-5, and IL-13 or into Th1 cells that secrete IL-2 and IFN-ץ. In the early-phase pathogenesis of $\mathrm{AD}$, the mast cells and basophils are activated by IgE-mediated pathway which is dependent on a Th2 phenotype of cytokine expression. IgE itself can regulate the expression of FceRI on these cells. When exposed to high levels of IgE, the mast cells and basophils express an increased number of FceRI receptors, which may cause them to become more sensitive to allergens, release more mediators at lower allergen concentrations, and/or release larger amounts of chemokines and other products in response to allergens [13]. In the present study, we found an increasing production of $\operatorname{IgE}$ in the skin lesions and serum in $\mathrm{AD}$ patients, compared with normal subjects.

In our study, we found the serum levels of IL-4, IL-5 and IL-13 in AD patients were increased, indicating their possible role in the AD pathogenesis. It has been suggested that epicutaneous sensitization of $\mathrm{AD}$ is a kind of Th2-mediated dermatitis [14]. IL-4 is a key Th2 cytokine important for Th2 cell differentiation, proliferation of B cells, IgE production, and eosinophil recruitment into the skin [15]. Analysis of CD4+ cord blood $\mathrm{T}$ cells indicate that elevated IL-4 levels in these cells are associated with an increased risk of AD [16]. Numerous in vitro studies have examined the effects of IL-4 on different cell types found in the skin [17]. Dermal fibroblasts isolated from patients with acute AD skin lesions are much more responsive to IL-4 in their ability to express eotaxin, another critical cytokine for eosinophil recruitment [18]. Normal human epidermal keratinocytes treated with IL-4 show increased expression of eotaxin, a key chemokine for eosinophil recruitment [19]. IL-5 is a critical cytokine for eosinophil development, survival and proliferation [20]. IL-5 plays a nonredundant role in the differentiation of eosinophils from bone marrow precursors [21]. Peripheral blood mononuclear cells (PBMCs) from patients with both extrinsic and intrinsic $\mathrm{AD}$ produce elevated levels of IL-5 [22].
Along with other cytokines, an elevated IL-5 level is detected in the skin and serum of AD patients and mouse models, which was positively correlated with IgE levels [23]. A previous report has shown that eosinophils are virtually absent in OVA-sentitized skin sites of IL-5-/- mice [24]. IL-13, a central type 2 cytokine, and its receptors have been shown to be produced by most immune cells [25]. It shares 20-25\% sequence homology and effector functions with IL-4, as both cytokines use IL-4Ralpha as a receptor subunit. IL-13 is involved in B-cell maturation, differentiation and IgE isotype switching [26]. It down-modulates macrophage activity, thereby inhibiting the production of proinflammatory cytokines and chemokines [27]. A skin specific IL-13 transgenic mouse model exhibits all the key features of AD, including pruritic dermatitis, elevated IgE levels, inflammatory cell infiltration into the skin, upregulation of other cytokines and chemokines, and skin fibrosis [28]. IL-13 treated keratinocytes also increase migration of CD4+CCR4+ skin homing T cells, thereby promoting an inflammatory response [29].

Chemokines play an important role in the recruitment of different subsets of inflammatory cells to allergic sites [30]. Chemokines and their receptors have specific tissue and cellular distribution patterns, leading to a selective inflammatory process in response to different stimuli. Chemokines are small, secreted proteins that can be subdivided into the $\mathrm{CC}$, CXC, $\mathrm{C}$ and $\mathrm{CX} 3 \mathrm{C}$ classes, according to their $\mathrm{NH}_{2}$-terminal cysteine-motifs. Eotaxin-1, one of the important chemotactic factors, promotes eosinophil infiltration, which is a major phenomenon of the late-phase reaction. Recent studies indicate a correlation between the serum levels of eotaxin and the activity of AD [31]. The level of Eotaxin, as well as the expression of CCR3, is higher in case of skin changes, with their further increase being observed in AD exacerbations [32].

As a recently identified human cytokine [33], CKLF1 is a potent chemoattractant for myopathies [34] and in arthritis [35], it is highly expressed on Th2 lymphocytes and plays an important role in the pathogenesis of asthma. The expression and function of CKLF1 are associated with asthma [36]. Mice treated with intradermal injection of CKLF1 or transgenic mice over-expressing CKLF1 develop allergic asthma. Up to date, there are no literatures reporting the expression of CKLF1 in skin tissue. In the present study, we firstly discovered that epidermal layer of the normal skin expresses CKLF1, indicating that it may participate in the basic immunologic function of the skin. However, there were fewer CKLF1 positive cells in dermis of the normal skin. Recent studies indicate that CKLF1 is highly expressed in many im- 
mune-mediated diseases [37]. In the present study, we explored the role of CKLF1 in AD, through determining the levels of CKLF1 in peripheral blood and the expression of CKLF1 and CKLF1 mRNA in skin lesions from $\mathrm{AD}$ patients. We found that serum CKLF1 levels in AD patients were significantly increased and positively correlated with serum Th2 cytokines IL-4, IL-5 and IL-13. We also found that skin CKLF1 protein and mRNA levels were significantly elevated in $\mathrm{AD}$ patients than that in the normal controls. Immunohistochemical staining results indicated that CKLF1 in cytoplasma and nuclei[38] was distributed on the keratinocytes of granular layer and basal layer of skin from $\mathrm{AD}$ patients as well as in normal controls. However, the skin lesions in AD patients showed more staining positive and intense cells in the epidermal than that in normal skin. CKLF1 staining positive cells in the upper dermis of the AD patients were detected in lymphocytes and other infiltrated cells. There were more CKLF1 positive staining cells infiltrated in the dermis of skin lesions in atopic dermatitis than those in normal skin. Our experimental results indicate that CKLF1 may participate in multiple immunological inflammation in AD. CKLF1 is a human cytokine that exhibits chemotactic activities. CC chemokine receptor 4 (CCR4), a member of G-protein coupled receptor family, is expressed on various cells and tissues, which is a specific marker for Th2-type lymphocytes. CKLF1 is also one of the ligands of CCR4 [39], which plays an important role in the occurrence and progression of $\mathrm{AD}$. As one of the important chemokine receptors in AD, CCR4 is highly expressed in most AD skin lesions [40], thus making it a potential target for treating inflammatory diseases.

In conclusion, the CKLF1 expression is increased in peripheral blood of $\mathrm{AD}$ patients, and there is a significant increase in the number of CKLF1 positive cells in AD skin lesions. Since CCR4 is chemokine receptor important to AD and CKLF1 is a functional ligand for human CCR4, we suggest that CKLF1 may have a functional role in $\mathrm{AD}$ development and therefore it may be a new target for the treatment of patients with $\mathrm{AD}$.

\section{Acknowledgement}

This study was supported by the National Natural Sciences Foundation of China (No. 81171498) and Beijing Municipal Science \& Technology Commission (No. Z111100067311040).

\section{Conflicting Interests}

We declare that we have no conflict of interest in relation to the publication of this article.

\section{References}

1. Butsch Kovacic M, Biagini Myers JM, Lindsey M, et al. The Greater Cincinnati Pediatric Clinic Repository: A Novel Framework for Childhood Asthma and Allergy Research. Pediatr Allergy Immunol Pulmonol. 2012; 25(2):104-113.

2. Guttman-Yassky E, Nograles KE, Krueger JG. Contrasting pathogenesis of atopic dermatitis and psoriasis--part I: clinical and pathologic concepts. J Allergy Clin Immunol. 2011; 127: 1110-1118.

3. Wen HJ, Chen PC, Chiang TL, et al. Predicting risk for early infantile atopic dermatitis by hereditary and environmental factors. Br J Dermatol. 2009; 161(5):1166-1172.

4. Banfield G, Watanabe H, Scadding G, et al. CC chemokine receptor 4 (CCR4) in human allergen-induced late nasal responses. Allergy. 2010; 65:1126-1133.

5. Willems LI, Ijzerman AP. Small molecule antagonists for chemokine CCR3 receptors. Med Res Rev. 2009; 30:778-817.

6. Owen CE. Immunoglobulin E: role in asthma and allergic disease: lessons from the clinic. Pharmacol Ther. 2007; 113:121-133.

7. Han W, Lou Y, Tang J, et al. Molecular cloning and characterization of chemokine-like factor 1 (CKLF1), a novel human cytokine with unique structure and potential chemotactic activity. Biochem J. 2001; 357:127-135.

8. Tan YX, Han WL, Chen YY, et al. Chemokine-like factor 1, a novel cytokine, contributes to airway damage, remodeling and pulmonary fibrosis. Chin Med J (Engl). 2004; 117:1123-1129.

9. Zheng $Y$, Guo C, Zhang $Y$, et al. Alleviation of murine allergic rhinitis by C19, a C-terminal peptide of chemokine-like factor 1 (CKLF1). Int Immunopharmacol. 2011; 11(12):2188-2193.

10. Hanifin JM, Rajka G. Diagnostic features of atopic dermatitis. Acta Derm Venerol. 1980; 92:44-47.

11. Guttman-Yassky E, Nograles KE, et al. Contrasting pathogenesis of atopic dermatitis and psoriasis--part II: immune cell subsets and therapeutic concepts. J Allergy Clin Immunol. 2011; 127(6):1420-1432.

12. Herberth G, Hinz D, Roder S, et al. Maternal immune status in pregnancy is related to offspring's immune responses and atopy risk. Allergy. 2011; 66(8):1065-1074.

13. Trangsrud AJ, Whitaker AL, Small RE. Intranasal corticosteroids for allergic rhinitis. Pharmacotherapy. 2002; 22:1458-1467.

14. Yalcindag A, He R, Laouini D,et al. The complement component C3 plays a critical role in both Th1 and Th2 responses to antigen. J Allergy Clin Immunol. 2006; 117(6):1455-1461.

15. Tokura Y. Extrinsic and intrinsic types of atopic dermatitis. J Dermatol Sci. 2010; 58(1):1-7.

16. Herberth G, Heinrich J, Röder S, et al. Reduced IFN-gamma- and enhanced IL-4-producing CD4+ cord blood T cells are associated with a higher risk for atopic dermatitis during the first $2 \mathrm{yr}$ of life. Pediatr Allergy Immunol. 2010; 21: 5-13.

17. Zhang D, Sanchez-Fueyo A, Kawamoto K, et al. Th1 to Th2 immune deviation facilitates, but does not cause, islet allograft tolerance in mice. Cytokine. 2010; 51(3):311-319.

18. Gahr N, Fölster-Holst R, Weichenthal M, et al. Dermal fibroblasts from acute inflamed atopic dermatitis lesions display increased eotaxin/CCL11 responsiveness to interleukin-4 stimulation. Br J Dermatol. 2011; 164:586-592.

19. Nishi N, Yamamoto S, Ou W, et al. Enhanced CCL26 production by IL-4 through IFN-gamma-induced upregulation of type 1 IL-4 receptor in keratinocytes. Biochem Biophys Res Commun. 2008; 376:234-240.

20. Takatsu K. Interleukin-5 and IL-5 receptor in health and diseases. Proc Jpn Acad Ser B Phys Biol Sci. 2011; 87(8):463-485.

21. Simon D, Braathen LR, Simon HU. Eosinophils and atopic dermatitis. Allergy. 2004; 59:561-570.

22. Simon D, Von Gunten S, Borelli S, et al. The interleukin-13 production by peripheral blood $\mathrm{T}$ cells from atopic dermatitis patients does not require CD2 costimulation. Int Arch Allergy Immunol. 2003; 132:148-155.

23. Jeong CW, Ahn KS, Rho NK, et al. Differential in vivo cytokine mRNA expression in lesional skin of intrinsic vs. extrinsic atopic dermatitis patients using semiquantitative RT-PCR. Clin Exp Allergy. 2003; 33:1717-1724

24. Spergel JM, Mizoguchi E, Oettgen H, et al. Roles of TH1 and TH2 cytokines in a murine model of allergic dermatitis. J Clin Invest. 1999; 103:1103-1111.

25. Akdis M, Burgler S, Crameri R, et al. Interleukins, from 1 to 37, and interferon- $\gamma$ : receptors, functions, and roles in diseases. J Allergy Clin Immunol. 2011; 127:701-721.

26. Jelinek DF. Regulation of B lymphocyte differentiation. Ann Allergy Asthma Immunol. 2000; 84(4):375-385. 
27. Akdis $M$, Burgler $S$, Crameri $R$, et al. Interleukins, from 1 to 37 , and interferon- $\gamma$ : receptors, functions, and roles in diseases. J Allergy Clin Immunol. 2011; 127:701-721.

28. Zheng T, Oh MH, Oh SY, et al. Transgenic expression of interleukin-13 in the skin induces a pruritic dermatitis and skin remodeling. J Invest Dermatol. 2009; 129:742-751.

29. Purwar R, Werfel T, Wittmann M. IL-13-stimulated human keratinocytes preferentially attract CD4+CCR4+ T cells: possible role in atopic dermatitis. J Invest Dermatol. 2006; 126:1043-1051.

30. Bisset LR, Schmid-Grendelmeier P. Chemokines and their receptors in the pathogenesis of allergic asthma: progress and perspective. Curr Opin Pulm Med. 2005; 11:35-42.

31. Kaburagi Y, Shimada Y, Nagaoka T, et al. Enhanced production of CC-chemokines (RANTES, MCP-1, MIP-1a, MIP-1b, and eotaxin) in patients with atopic dermatitis. Arch Dermatol Res. 2001; 293:350-355.

32. Yawalkar N, Uguccioni M, Scharer J, et al. Enhanced expression of eotaxin and CCR3 in atopic dermatitis. J Invest Dermatol. 1999; 113:43-48.

33. Han W, Lou Y, Tang J, et al. Molecular cloning and characterization of chemokine-like factor 1 (CKLF1), a novel human cytokine with unique structure and potential chemotactic activity. Biochem J. 2001; 357:127-135.

34. Chowdhury MH, Nagai A, Terashima M, et al. Chemokine-like factor expression in the idiopathic inflammatory myopathies. Acta Neurol Scand. 2008; 118:106-114.

35. Rioja I, Clayton CL, Graham SJ, et al. Gene expression profiles in the rat streptococcal cell wall-induced arthritis model identified using microarray analysis. Arthritis Res Ther. 2005; 7:101-117.

36. Tian L, Li W, Wang J, Zhang Y, Zheng Y, Qi H, Guo X, Zhang Y, Ma D, Shen H, Wang Y. The CKLF1-C19 peptide attenuates allergic lung inflammation by inhibiting CCR3- and CCR4-mediated chemotaxis in a mouse model of asthma. Allergy. 2011; 66(2):287-97.

37. Rojas Ramos E, Martínez Jiménez N, Reyes Salinas A. The Th2 theory in allergy: present and future directions. Rev Alerg Mex. 2003; 50(2):64-70.

38. Jing Huang, Li Zhang, Wei Liu, Qinyuan Liao, Taiping Shi, Lin Xiao, Fanlei Hu, Xiaoyan Qiu. CCDC134 interacts with hADA2a and functions as a regulator of hADA2a in acetyltransferase activity, DNA damage-induced apoptosis and cell cycle arrest. Histochem Cell Biol. 2012; 138(1):41-55.

39. Wang $Y$, Zhang $Y$, Yang $X$, et al. Chemokine-like factor 1 is a functional ligand for CC chemokine receptor 4 (CCR4). Life Sci. 2006; 78:614-621.

40. Nakatani T, Kaburagi Y, Shimada Y, et al. CCR4 memory CD4+ T lymphocytes are increased in peripheral blood and lesional skin from patients with atopic dermatitis. J Allergy Clin Immunol. 2001; 107:353-358. 